\title{
Influence of Substrate on Crystallographic Quality of AlGaN/GaN HEMT Structures Grown by Plasma-Assisted MBE
}

\author{
A. Wierzbicka*, Z.R. Żytkiewicz, M. Sobańska, K. KŁosek, E. Łusakowska \\ Institute of Physics Polish Academy of Sciences, al. Lotników 32/46, 02-668 Warsaw, Poland
}

\begin{abstract}
Results of characterization of $\mathrm{AlGaN} / \mathrm{GaN}$ high electron mobility transistor (HEMT) structures grown by plasma-assisted molecular beam epitaxy (PAMBE) are reported. High resolution X-ray diffraction (HRXRD) and X-ray reflectivity (XRR) were applied to show that structural properties of the AlGaN/GaN layers strongly depend on the substrate used for growth. It has been found that an additional $10 \mu \mathrm{m}$ thick HVPE GaN layer grown on a commercial GaN/sapphire substrate significantly improves structural quality of AlGaN layer. However, the best structural parameters have been obtained for the HEMT sample grown on free-standing HVPE bulk GaN substrate.
\end{abstract}

PACS: 61.05.cp, 61.05.cm, 81.15.Hi, 68.55.-a

\section{Introduction}

$\mathrm{AlGaN} / \mathrm{GaN}$ high electron mobility transistors (HEMT) are promising devices for high frequency and high power electronic devices. Since bulk GaN substrates are not readily available on an industrial scale, they are usually grown on alternative substrates like sapphire or silicon. In that case, however, a lattice mismatch with the substrate leads to large strain and its relaxation via defect formation in the layers, which in turn affects the properties of devices. In particular, it is well known that mobility of two-dimensional electron gas (2DEG) may be seriously limited by dislocations, interface roughness or deformation potential [1].

In this work the influence of substrate structure on the crystallographic quality of AlGaN/GaN HEMT grown by plasma-assisted molecular beam epitaxy (PAMBE) has been examined. Commercially available $\mathrm{GaN} /$ sapphire substrates, as received and regrown by additional $10 \mu \mathrm{m}$ thick HVPE GaN, as well as free-standing HVPE bulk GaN substrates were used for the growth. High resolution X-ray diffraction (HRXRD) and X-ray reflectivity (XRR) were applied to measure basic structural properties of HEMTs as lattice parameters, strain distribution in the samples, thicknesses of the layers and roughness of their interfaces. We have found that regrowth of commercial GaN/sapphire substrates with $10 \mu \mathrm{m}$ thick HVPE GaN layer significantly improves the structural quality of AlGaN layer. However, the best structural parameters have been obtained for the HEMT samples grown on HVPE bulk GaN substrate.

\section{Experiment}

AlGaN/GaN HEMTs were grown using PAMBE technique in Riber Compact 21 system with elemental sources

* corresponding author; e-mail: wierzbicka@ifpan.edu.pl of $\mathrm{Al}, \mathrm{Ga}, \mathrm{In}, \mathrm{Si}$ and $\mathrm{Mg}$. Active nitrogen was supplied from an Addon RF plasma source. Figure 1 schematically shows cross-sections of HEMT structures studied in this work. All of them contain undoped GaN buffer, $20 \mathrm{~nm}$ thick AlGaN layer with composition of $\sim 20 \% \mathrm{Al}$ and $3 \mathrm{~nm}$ thick GaN cap. However, the samples differ in the substrates used. Samples 1 and 2 were grown on commercially available $3 \mu \mathrm{m}$ thick GaN:Fe/sapphire substrates grown by metalorganic vapor phase epitaxy (see Fig. 1a-b). In the Sample 2 an additional $10 \mu \mathrm{m}$ thick GaN layer was deposited by hydride vapor phase epitaxy (HVPE) on the template. The third sample (Fig. 1c) was grown on a free-standing bulk GaN substrate grown by HVPE.

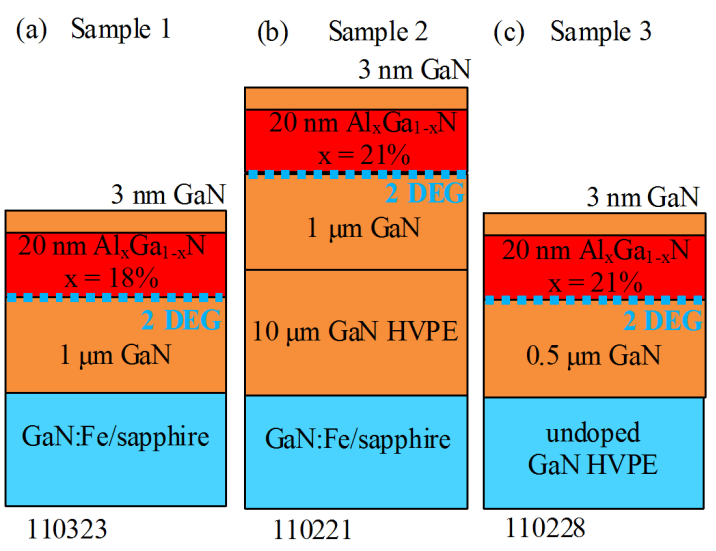

Fig. 1. Schematic cross sections of the investigated $\mathrm{Al}_{x} \mathrm{Ga}_{1-x} \mathrm{~N} / \mathrm{GaN}$ HEMT structures.

Structural properties of the layers were analyzed by HRXRD and XRR techniques. High resolution X-ray measurements were performed by Panalytical X'Pert Pro MRD diffractometer equipped with hybrid two-bounce Ge (220) monochromator and triple bounce Ge (220) an- 
alyzer in front of the detector. For each sample GaN 0002 symmetric reflection in $\omega$ and $2 \theta / \omega$ mode was measured. It allows to measure values of lattice parameters $c$ and lattice deformations in (0001) direction. Next GaN $\overline{11} 24$ asymmetric reflection in $\omega$ and $2 \theta / \omega$ mode was studied to get the lattice parameters $a$ and in-plane distortion. Biaxial in-plane stress that exists in hexagonal GaN film on sapphire substrate was taken into account in the analysis. The composition of the AlGaN layer was obtained by Vegard's law (VL). X-ray diffraction $2 \theta / \omega$ profiles were simulated by commercial Panalytical software based on the Takagi-Taupin theory [2-4]. It is important to mention that in order to eliminate influence of sample bowing on the FWHM values of diffraction curves the following recipe was used: each diffraction curve was measured for a series of widths of incident X-ray beam. Then, the dependence of FWHM versus the width of the beam was plotted and fitted with the linear function. The values of FWHM reported in this work are those obtained under the "zero width" X-ray beam conditions.

$\mathrm{X}$-ray reflectivity measurements were applied to determine the thicknesses of the individual layers and the root-mean square (RMS) roughness of their interfaces and of the upper surface. XRR is proved to be effective to estimate structural features in heteroepitaxial layers [5]. In this work X-ray reflectivity measurements were performed with the use of Panalytical X'Pert Pro MRD diffractometer, a Parallel Plate Collimator with $0.4 \mathrm{rad}$ Soller slits and $0.18 \mathrm{deg}$ divergence slit was applied in front of detector. The advantage of this geometry is that it can be used at grazing incidence to enhance the scattering from thin films. The parallel-plate collimator enables an angular selection of the expanded beam caused by the low angle of incidence beam on the sample. $\mathrm{X}$ ray reflectivity curves were simulated using commercial Panalytical software based on Parratt theory [6]. Finally, the surface morphology of the samples was analyzed by atomic force microscopy (AFM) and compared with values obtained by means of the XRR technique.

\section{Results and discussion}

Figure 2 shows $2 \theta / \omega$ X-ray diffraction curves of the HEMT structures (blue lines) measured in triple axis geometry (with an analyzer). The symmetrical $0002 \mathrm{CuK}_{\alpha 1}$ reflection was measured. The scans contain the main peak from GaN layers that are present in the sample. Their angular position is similar for the Sample 1 and 2, whereas for the Sample 3 it is shifted by $0.039^{\circ}$. Due to the penetration of the crystal by the incident X-ray beam, estimated for this reflection to be $\sim 15 \mu \mathrm{m}$, diffraction peak shows the information from the sample that is averaged over some depth. Therefore, the values of $c$ lattice parameters of the GaN layer are similar for Samples 1 and 2, but are different from the value obtained for the Sample 3. Diffraction curves also contain the AlGaN peaks, the intensity of which is significantly reduced due to the smaller thickness of that layer. From these data the calculations of lattice parameters $c$ were made. Moreover, the performed simulations of the diffraction data (red curves in Fig. 2) allowed the determination of thicknesses of the individual GaN and AlGaN layers as well as the composition of ternary layers. All these results are collected in Table I. As seen, the Al content in AlGaN layer is equal to $18 \%$ for Sample 1 and $21 \%$ for Samples 2 and 3, while the thickness of AlGaN is equal to $\sim 20$ $\mathrm{nm}$. These results are close to the designed values.

\section{TABLE I}

Lattice parameters, thicknesses and compositions of layers in $\mathrm{AlGaN} / \mathrm{GaN}$ HEMT samples as obtained from HRXRD measurements

\begin{tabular}{|c|c|c|c|c|c|c|}
\hline & \multicolumn{3}{|c|}{$\mathrm{GaN}$} & \multicolumn{3}{|c|}{$\mathrm{Al}_{x} \mathrm{Ga}_{1-x} \mathrm{~N}$} \\
\hline Sample & 1 & 2 & 3 & 1 & 2 & 3 \\
\hline \multicolumn{7}{|l|}{ Parameters } \\
\hline$c \pm 0.002[\AA]$ & 5.1874 & 5.1892 & 5.1850 & 5.1442 & 5.1489 & 5.1485 \\
\hline$a \pm 0.008[\AA]$ & 3.1852 & 3.1839 & 3.1900 & 3.1862 & 3.1725 & 3.1769 \\
\hline $\begin{array}{l}\text { composition } \\
\pm 2 \% \text { (VL) }\end{array}$ & & & & 16 & 19 & 18 \\
\hline $\begin{array}{l}\text { composition } \\
\pm 1 \% \text { (from } \\
\text { simulation } \\
\text { of } 2 \theta / \omega \text { scan) }\end{array}$ & & & & 18 & 21 & 21 \\
\hline \multicolumn{7}{|c|}{ Thickness $\pm 1[\mathrm{~nm}]$ (from simulation of $2 \theta / \omega$ scan) } \\
\hline $\begin{array}{l}\text { Sample } \\
\text { Layers }\end{array}$ & \multicolumn{2}{|c|}{1} & \multicolumn{2}{|c|}{2} & \multicolumn{2}{|c|}{3} \\
\hline GaN cap & \multirow{2}{*}{\multicolumn{2}{|c|}{$\begin{array}{c}3 \\
20\end{array}$}} & \multicolumn{2}{|c|}{3} & \multicolumn{2}{|c|}{3} \\
\hline $\mathrm{AlGaN}$ & & & \multicolumn{2}{|c|}{20} & \multicolumn{2}{|c|}{21} \\
\hline $\mathrm{GaN}$ & \multirow{2}{*}{\multicolumn{2}{|c|}{$\begin{array}{c}20 \\
1000\end{array}$}} & \multicolumn{2}{|c|}{1000} & \multirow{2}{*}{\multicolumn{2}{|c|}{500}} \\
\hline $\mathrm{GaN}$ & & & & 000 & & \\
\hline
\end{tabular}

In order to determine the deformation of the $\mathrm{AlGaN}$ lattice in the layers deposited on different GaN substrates the lattice parameter $a$ was measured. Figure 3 shows reciprocal space maps of the $\overline{11} 24$ reflection of a $\mathrm{Al}_{x} \mathrm{Ga}_{1-x} \mathrm{~N} / \mathrm{GaN}$ HEMTs structures. As seen the broadest diffracted signal for the GaN layers is observed for Sample 1 (Fig. 3a) and the narrowest for sample 3. It is obvious that the homoepitaxial layer, i.e. the sample 3 grown on bulk GaN substrate, have the smallest full width at half maximum $\left(\mathrm{FWHM}=0.024^{\circ}\right)$, which indicates the best structural quality. For Samples 1 and 2 the FWHM are equal to $0.056^{\circ}$ and $0.043^{\circ}$, respectively. It is worth noting, that in Sample 2 an additional $10 \mu \mathrm{m}$ thick GaN HVPE layer significantly improves the quality of GaN structure in comparison to sample 1. Similar behavior is observed for the AlGaN layers in HEMT structures. Values of FWHM for asymmetrical $\overline{11} 24$ reflection are equal to $0.138^{\circ}, 0.068^{\circ}$ and $0.030^{\circ}$ for the first, second and third sample, respectively. This indicates significant improvement of overall structural quality of HEMTs when thickness of GaN substrate increases.

Reciprocal space maps of the $\overline{11} 24$ reflection (Fig. 3) shows that for all samples the reciprocal lattice point corresponding to AlGaN barrier is located just above 
the GaN diffracted signal. It indicates pseudomorphic growth of AlGaN. Moreover, the values of lattice parameters $a$ were determined from these maps (see Table I). Finally, the $c / a$ ratio was calculated. As it is known, the theoretical value of $c / a$ ratio for the wurzite structures is equal to 1.633 and only structures with $c / a \leq 1.633$ are stable [7]. For the HEMT structures studied in this work the values of $c / a$ ratio equal to $1.6256,1.6257$ and 1.6258 for $\mathrm{GaN}$ and 1.6219, 1.6211 and $1.6214( \pm 0.0002)$ for the AlGaN barriers for the first, second and third sample, respectively. These values are very close to that reported for GaN bulk crystals [8, 9].

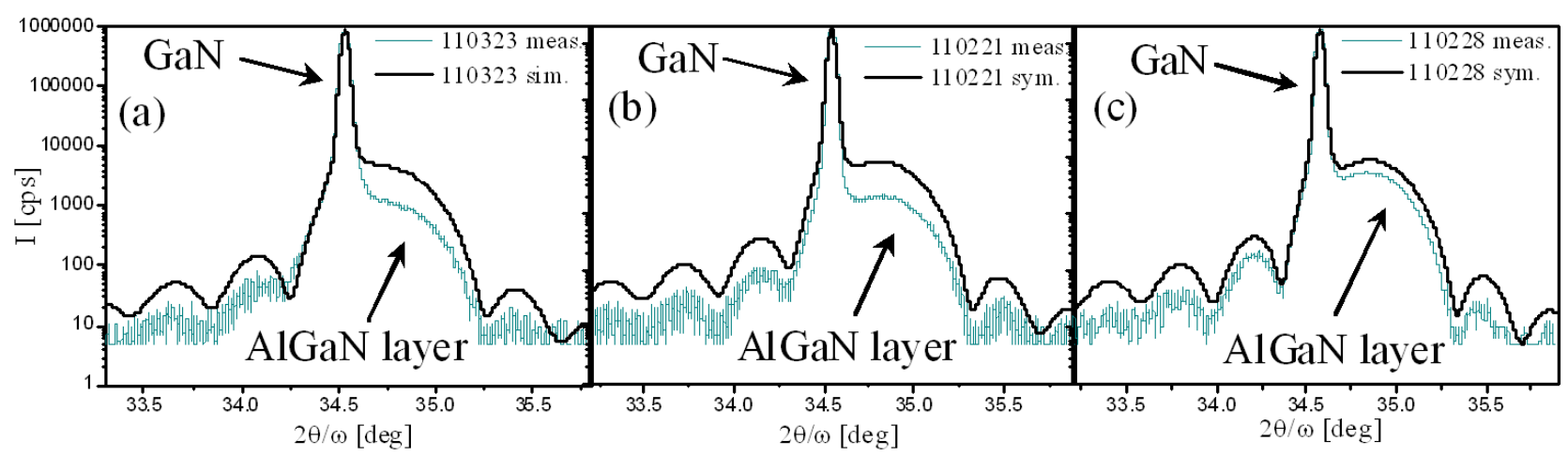

Fig. 2. Experimental and simulated diffraction curves $(2 \theta / \omega)$ of $00.2 \mathrm{CuK}_{\alpha 1}$ reflection from the $\mathrm{Al}_{x} \mathrm{Ga}_{1-x} \mathrm{~N} / \mathrm{GaN}$ HEMTs structures. (a) (b) and (c) graphs correspond to Samples 1, 2 and 3, respectively.
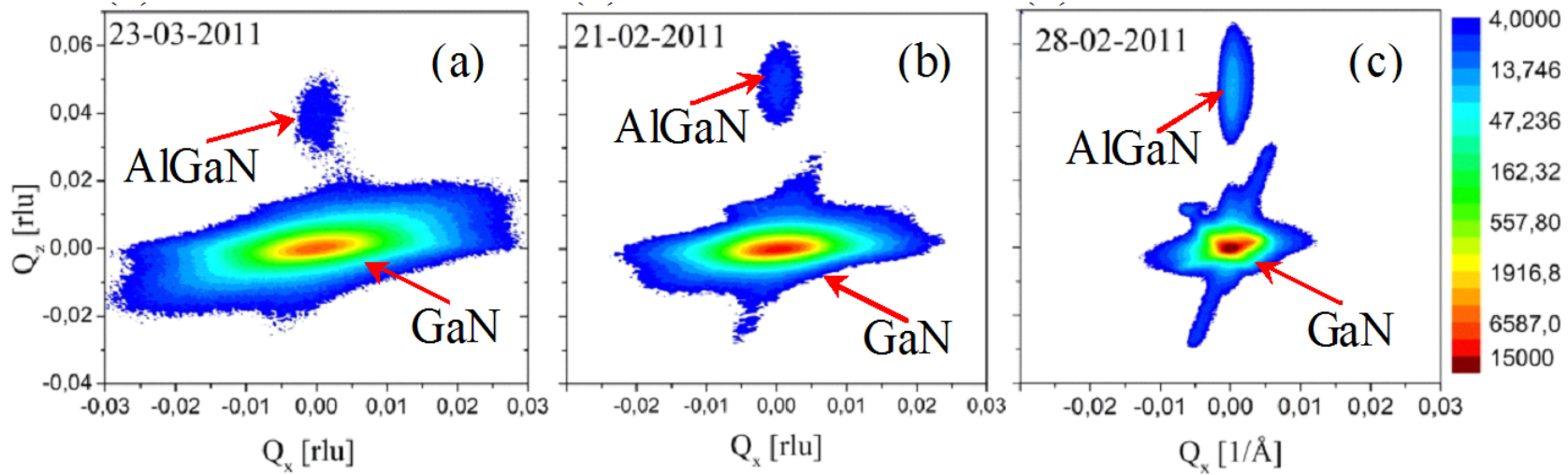

Fig. 3. Reciprocal space maps of the asymmetric $\overline{11} 24$ reflection of a $\mathrm{Al}_{x} \mathrm{Ga}_{1-x} \mathrm{~N} / \mathrm{GaN}$ HEMTs structures. Axes are marked in $\lambda / 2 d$ units $\left(\lambda=1.5406\right.$ - wavelength, $d$ - lattice spacing of (11.4)). $\mathrm{Q}_{x}$ axis is in $\langle 11.0\rangle$ direction (parallel to the surface), $\mathrm{Q}_{z}$ axis is in $\langle 00.1\rangle$ direction (perpendicular to the surface).

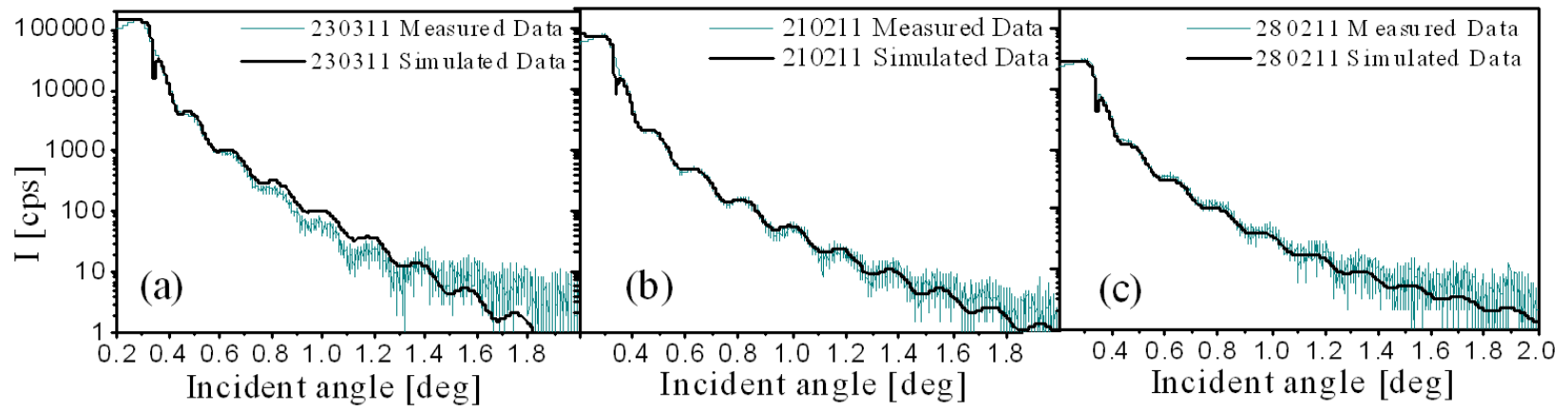

Fig. 4. Experimental and simulated X-ray reflectivity curves of a $\mathrm{Al}_{x} \mathrm{Ga}_{1-x} \mathrm{~N} / \mathrm{GaN}$ HEMTs structures for Samples $1(\mathrm{a}), 2(\mathrm{~b})$ and $3(\mathrm{c})$.

Figures $4 \mathrm{a}-\mathrm{c}$ show results of analysis of three HEMT samples by means of the X-ray reflectivity technique. Oscillations on the reflectivity curves, which are due to the $\mathrm{AlGaN} / \mathrm{GaN}$ interfaces and commonly known as Kiessig fringes [8], are clearly visible in these profiles. Their presence indicates smooth surfaces and allows to determine the thicknesses of the layers. The values of critical angles for all samples studied are the same, which indicates the same mass densities, i.e. similar $\mathrm{Al}$ content, in the studied samples. Experimental curves were simulated using commercial Panalytical software, in which the fitting parameters were the thickness and mass densities of the 
layers as well as the roughness of their surfaces. Results of XRR simulations are collected in Table II. As seen, in all samples the thicknesses of GaN cap and AlGaN agree well with those measured by HRXRD and are very close to values designed by the growers. It is worth noting that in all the samples the roughness of the $\mathrm{AlGaN} / \mathrm{GaN}$ interface is quite small. This is of prime importance for HEMT structures since the channel with high mobility two-dimensional electron gas is located just at this interface. Also the upper surfaces of the structures are very smooth. The RMS values from XRR simulations are equal to $6 \AA, 4 \AA$ and $1 \AA$ for the first, second and third sample, respectively. These values are slightly different than those obtained by AFM (12 $\AA, 9 \AA$ and 8 $\AA$, respectively), which might be caused by different areas probed by the two techniques, but improvement of surface quality from Sample 1 to Sample 3 is preserved.

TABLE II

Thicknesses of the layers and roughness of their interfaces obtained from XRR for Samples 1, 2 and 3

\begin{tabular}{l|c|c|c|c|c|c}
\hline \hline & \multicolumn{3}{|c|}{$\begin{array}{c}\text { Thickness } \\
\pm 0.1[\mathrm{~nm}]\end{array}$} & \multicolumn{3}{c}{$\begin{array}{c}\text { Roughness of upper } \\
\text { surface } \pm 0.1[\mathrm{~nm}]\end{array}$} \\
\hline \multicolumn{1}{c}{ Samples } & 1 & 2 & 3 & 1 & 2 & 3 \\
Layers & & & & & & \\
\hline GaN cap & 2.1 & 3 & 3.1 & 0.6 & 0.4 & 0.1 \\
AlGaN & 20.8 & 20 & 20.4 & 0.9 & 1 & 1 \\
GaN & 967.9 & 1050 & 464.7 & 0.5 & 0.4 & 0.3 \\
GaN & & 9749.5 & & & 0.1 &
\end{tabular}

\section{Summary and conclusions}

Influence of substrate structure on crystallographic quality of AlGaN/GaN HEMTs grown by PAMBE has been examined. HRXRD technique was used to prove the pseudomorphic growth of the AlGaN layers and to measure their composition, lattice parameters and strain distribution in the samples. We have found that an additional $10 \mu \mathrm{m}$ thick HVPE GaN layer grown on the commercial GaN/sapphire substrates significantly improves the structural quality of $\mathrm{AlGaN}$ layer. However, the best structural parameters have been obtained for the HEMT sample grown on a HVPE bulk GaN substrate.

The X-ray reflectivity was also used to measure thicknesses of layers and to evaluate roughness of their interfaces. In particular, thicknesses of GaN cap and AlGaN obtained from XRR simulations agree well with those measured by HRXRD and are very close to values designed by the growers. High quality of the AlGaN/GaN interfaces, especially in the sample grown on bulk GaN substrate, is found. XRR shows also decrease of GaN cap surface roughness when the GaN/sapphire substrate is regrown by HVPE. Even better surface quality is found in HEMT structures grown on bulk GaN substrate. These results agree with roughness data obtained by AFM on the same samples.

\section{Acknowledgments}

The authors are grateful to B. Łucznik for supplying the HVPE GaN substrate. HVPE-regrown $\mathrm{GaN} /$ sapphire substrates have been received from EPFL, Lausanne. This work was partially supported by the European Union within European Regional Development Found, through grant Innovative Economy POIG.01.01.02-00-008/08 NanoBiom.

\section{References}

[1] P.C. Khai, T. Suzue, Y. Sakai, S. Lawrence Selvaraj, T. Egawa, T. Jimbo, Phys. Stat. Sol. C 7, 1949 (2010).

[2] S. Takagi, Acta Crystallogr. 15, 1311 (1962).

[3] S. Takagi, J. Phys. Soc. Japan 26, 1239 (1969).

[4] D. Taupin, Bull. Soc. Franc. Mineral. Cristall. 87, 469 (1964).

[5] S. Corekci, M.K. Ozturk, B. Akaoglu, M. Cakmak, S. Ozcelik, E. Ozbay, J. Appl. Phys. 101, 123502 (2007).

[6] L.G. Parratt, Phys. Rev. 95, 359 (1954).

[7] M.A. Moram, M.E. Vickers, Rep. Prog. Phys. 72, 036502 (2009).

[8] M. Leszczynski, T. Suski, J. Domagala, P. Prystawko in: Properties, processing and applications of gallium nitride and related semiconductors, INSPEC, London (1999).

[9] T. Deguchi, D. Ichiryu, K. Toshikawa, K. Sekiguchi, T. Sota, R. Matsuo, T. Azuhata, J. Appl. Phys. 86, 1860 (1999). 